\title{
Don Quijote, humor y gestos
}

\author{
Guillemette Bolens*
}

\begin{abstract}
Resumen
Este artículo trata del análisis kinésico, es decir de un método de análisis de los gestos y de los movimientos en la literatura. Propone desarrollar los medios de dar cuenta de un humor que proviene de las dinámicas corporales en interacción, tal y como se actualizan en la lectura de una obra literaria. Cuando las vivencias corporales se encuentran separadas por siglos y múltiples variaciones culturales, ¿cómo puede la literatura hacernos comprender y sentir las sensaciones dinámicas relatadas por los escritores hasta el punto de hacernos reír? Por encima de las brechas históricas innegables que nos obligan a una gran prudencia, ¿existen aspectos compartidos que nos permiten comprender las vivencias corporales de las que habla un escritor como Cervantes a través de los movimientos que describe en sus relatos? Este artículo trata de la cuestión específica del tono corporal integrado a perspectivas narratológicas y cognitivas.
\end{abstract}

Palabras clave: Don Quijote; Tristam Shandy; Geoffrey Chaucer; humor; cognición; tonus; análisis kinésico.

\section{Title: Don Quijote, humour and gestures}

\footnotetext{
Abstract:

The present article is about a method of analysis of gestures and movements in literature called kinesic analysis. Its purpose is to develop ways of accounting for a type of humour elicited by narrated corporeal dynamics in interaction, as perceived in the cognitive act of reading literature. When centuries and multiple cultural variations separate corporeal experiences, how does literature enable us to grasp the dynamic sensations narrated by authors to the point of making us laugh? Beyond the undeniable historical divide that calls for the greatest caution, are there shared aspects that allow us to understand the type of embodied experience that an author such as Cervantes conveys through the movements of his writ-

*Universidad de Ginebra. Guillemette.Bolens@unige.ch 
ing? In a perspective that is both «narratological» and cognitive, this article provides an approach that takes into account the cognitive process called perceptual simulation, paying a particular attention to the sensorimotor aspect of tonicity.

Key Words: Don Quixote; Tristram Shandy; Geoffrey Chaucer; humour; cognition; tonus; kinesic analysis.

A la memoria de Jocelyne Leal Su alegría y su luz nos habitan

«pero yo, inclinado de mi estrella» Cervantes, Don Quijote

«Quienes no tienen poder sobre la historia que domina sus vidas, poder para volverla a contar, para repensarla, de-construirla, bromear sobre ella y cambiarla con el paso del tiempo, son verdaderamente impotentes porque no pueden imaginar nuevos pensamientos $\rangle^{1}$

Salman Rushdie

Ser capaz de concebir nuevos pensamientos, según los términos de Salman Rushdie, consiste en poder movilizar de manera diferente el relato dominante de su propia vida. Saber reír es lo que Miguel de Cervantes, Laurence Sterne y otros genios literarios como Geoffrey Chaucer y François Rabelais han sabido transmitirnos para que podamos entender la importancia de esos relatos que hacen de nosotros lo que somos y, a partir de ellos, poder crear de nuevo.

En autores como Chaucer, Rabelais, Cervantes y Sterne, el humor desempeña un papel fundamental. Implica una inteligencia y una libertad que nos acercan a ellos y a los demás. El humor es un fenómeno complejo en la literatura pues reúne una serie de registros que operan simultáneamente. Para entender el humor de un gesto recurrimos a una forma de inteligencia que descansa en nuestro saber corporal. Esta inteligencia es kinésica, es decir, procede de un saber que se refiere a la sensorimotricidad, a nuestra capacidad de percibir, sentir, actuar y movernos. La inteligencia kinésica nos permite comprender los movimientos del otro en situación real. Pero también la movilizamos cuando leemos un relato que describe movimientos y gestos ${ }^{2}$.

1. Rushdie (1991). Extracto de un discurso pronunciado en la Universidad de Columbia el 11 de diciembre de 1991.

2. El concepto de inteligencia kinésica reenvía a nuestra capacidad de comprender pre-reflexivamente los movimientos corporales (en el otro y en las obras de arte, cualquiera que sea el medio empleado). Cf. Bolens (2008 y 2012a). Propongo en esta obra un desarrollo del concepto de inteligencia kinésica tal y como estableció en primer lugar Ellen Spolsky. 
Al mismo tiempo que explotamos nuestra inteligencia kinésica para comprender un gesto narrado, el relato que leemos puede apelar a nuestro conocimiento de otros textos y a su peculiar forma de describir los gestos y los movimientos corporales. Incluso puede decirse que el relato existe como respuesta a un texto precedente y a su manera de describir el cuerpo en sus movimientos. Se trata entonces de una relación hipertextual entre los textos: un texto ha provocado la escritura de otro nuevo.

El propósito de mi libro L'Humour et le savoir des corps: Don Quichote,, Tristram Shandy et le rire du lecteur (del que tomo aquí una parte de la introducción) es observar los puntos en que se cruzan el saber sensorimotor y la resonancia hipertextual: entre nuestro conocimiento de la realidad vivida del cuerpo y las milenarias y múltiples maneras de dar vida a esta realidad mediante el relato de los gestos y las acciones humanas.

Cuando las vivencias corporales se encuentran separadas por el paso de los siglos, y por variaciones epistemológicas y culturales múltiples, podemos preguntarnos por la forma en que la literatura nos hace comprender y vivir las sensaciones dinámicas que, narradas por los autores, consiguen hacernos reír. Más allá de las brechas históricas innegables que nos obligan a ser muy prudentes, ¿existen aspectos compartidos que nos permiten comprender las vivencias corporales de las que hablan Chaucer, Rabelais, Cervantes y Sterne a través de los movimientos que describen en sus narraciones ${ }^{3} \mathrm{Y}$ cuando esos aspectos aparecen en pasajes humorísticos, ¿cómo tratarlos?; ¿qué nos hace pensar que son humorísticos?; ¿cómo explicar hoy, en el siglo XXI, que un texto escrito en los siglos XVI, XVII o XVIII nos hace reír?

La relación humorística es una relación estética y, por tanto, subjetiva. Para Gérard Genette,

[S]i lo que hace reír a unos no hace necesariamente reír a otros es porque la risa no se funda en ningún concepto, en ningún principio general que estipule una relación universal y necesaria entre un determinado aspecto del objeto y el sentimiento de lo cómico. La relación entre el objeto y su efecto sólo es más o menos frecuente, y por consiguiente, probable. No hay objeto que sea cómico por sí mismo y que haga reír por esencia: no es el objeto el que hace reír: es el reír el que hace calificar de cómico al mismo objeto que, aparentemente, es su causa ${ }^{4}$.

A la lectura de un texto, el sentimiento de humor procede de la recepción subjetiva de los lectores. A partir de ahí, si los lectores de Don Quijote y de

3. En Bolens (2000) describo como la literatura puede permitirnos encontrar el rastro de lógicas corporales extremadamente diferentes de las que dominan al lector en el momento de la lectura. Ser conscientes de nuestros «a priori» tácitos en lo que se refiere a la corporeidad es la condición sine qua non de una investigación válida en la historia del cuerpo, condición sin la cual nos prohibimos el acceso a las lógicas corporales, a menudo extrañas, que confieren su coherencia a obras tan importantes como La Iliada, Beowulf o el Lancelot de Chrétien de Troyes.

4. Genette (2012: 453-454). 
Tristram Shandy ríen (como me sucedió a mí), ¿qué es lo que me permite rendir cuentas con precisión? En el marco general de la problemática del humor, mi libro somete a prueba dos nuevas pistas de análisis kinésico, la del tempo y la del tono, integradas en perspectivas narratológicas, cognitivas y socio-históricas.

En una obra científica de cognición comparada, los editores Edward Wasserman y Thomas Zentall inauguran su introducción tomando como ejemplo un acontecimiento A comunicado de dos maneras contrastadas. Versión A1: "Al principio, el impulso es débil; un deseo lánguido y vago se hace sentir acompañado de una dulce agitación. Más tarde crece, irresistible, la fuerza de la urgencia; vuelve bruscamente su cabeza y se lanza a través del suelo irregular hasta acariciar el objeto de su afecto con un placer extremo». Versión A2: «Una pieza metálica es atraída por un imán» ${ }^{5}$.

Al escoger este ejemplo, mi propósito consiste en evidenciar, de entrada y antes de volver sobre ello más detenidamente, nuestra presteza cognitiva para inferir un tono muscular en los movimientos de un cuerpo. La posibilidad de una acción autónoma sugiere un sistema locomotor capaz de variaciones tónicas de contracción y de-contracción musculares mediante las cuales se efectúan los movimientos. Si hay humor en el contraste flagrante que separa la versión A1 «overdramatized», de la versión A2 «objective, materialistic, and mechanistic» ${ }^{6}$ es porque nos sorprende que la tonicidad se haya situado allí donde sabemos, retrospectivamente, que está ausente, es decir, en el metal. Más que declarar que A1 carece de sentido y que A2 es científica ${ }^{7}$, subrayemos el hecho de que nuestra comprensión de la versión A1 implicó la inferencia implícita de una modificación del estado tónico del cuerpo descrito, pues nos pareció que debía estar vivo al estar dotado de deseos, de impulsos, de intenciones y de movimientos autónomos. Nuestra inferencia se realiza espontáneamente como reacción a lo que leemos ${ }^{8}$, ya que las modificaciones tónicas se sitúan en el centro de nuestra experiencia de lo viviente y las inferimos muy rápidamente con el tratamiento cognitivo de las informaciones motrices. Es la sorpresa que resentimos cuando nos damos cuenta de nuestro error (el movimiento tiene lugar entre objetos metálicos) la que nos permite tomar conciencia retroactivamente de nuestra tendencia a inferir un tono cuando pensamos el movimiento viviente. Producimos esta inferencia de manera pre-reflexiva mediante una percepción simulada cognitivamente, lo que no implica recurrir al concepto lexicalizado del tono.

Explicaré el proceso cognitivo de las simulaciones perceptivas tras el análisis de dos pasajes en los que Cervantes y Sterne introducen la sensorimotricidad en el relato cuando explotan la capacidad de sus lectores para inferir variaciones tónicas y, a través de ellas, los estados mentales y afectivos más finos y más cómicos de sus personajes. Pero antes es conveniente considerar dos ejemplos visuales que

5. Wasserman y Zentall (2006: 3).

6. Wasserman y Zentall (2006: 3).

7. Wasserman y Zentall (2006: 3). Estas cualificaciones son perfectamente comprensibles en la introducción de Wasserman y Zentall habida cuenta de los objetivos de su argumentación.

8. Por lo que se refiere a los últimos avances, los más interesantes conciernen al vínculo entre literatura y cognición, ver Cave (2016). 
permiten comprender rápidamente la naturaleza de las simulaciones perceptivas. El primer ejemplo es un cuadro de Chardin en el que un joven insufla una pompa de jabón con una paja (ver Figura 1). Nuestra comprensión de la forma circular y transparente que llama la atención de los dos personajes implica que simulamos perceptivamente la sustancia específica de este objeto. Si no activamos cognitivamente esta simulación (consciente o pre-conscientemente), la forma redonda que pende del extremo de un tubo fino no adquiere el sentido esperado por el artista, es decir, el de una pompa de jabón. A partir del momento en que nos adentramos en esta simulación perceptiva comprendemos que la sustancia de esta forma implica que el más mínimo contacto haría estallar la pompa y ello a pesar de que, en realidad, lo que miramos es una imagen hecha de pigmentos estáticos aplicados a una tela.

\section{FIGURA 1}

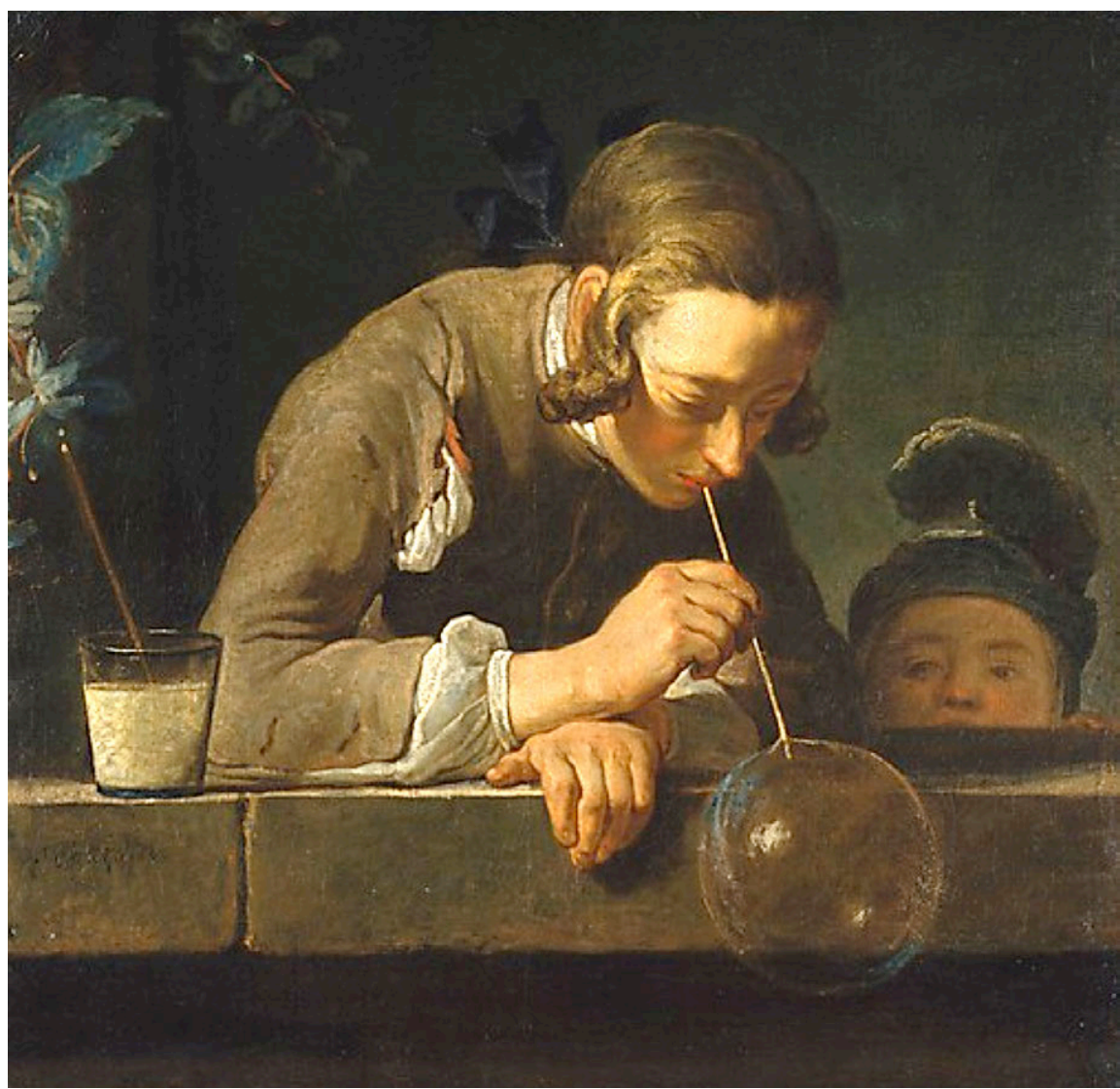

Jean Baptiste Siméon Chardin (1699-1779)

La bulle de savon, 1733-34

(The Metropolitan Museum of Art, Nueva York)

https://commons.wikimedia.org/wiki/File:Jean-Baptiste_Sim\%C3\%A9on_Chardin_022.jpg 
Mi segundo ejemplo es un dibujo de Jacques Sempé, uno de los mejores dibujantes franceses de este siglo y del pasado (ver Figuras 2 y 3). La simulación perceptiva que produce el espectador gracias a la comprensión de este dibujo implica al tono muscular. Comprendemos por simulación perceptiva el acontecimiento tónico de gran amplitud que se produce en el pequeño cuerpo del niño que intenta ejercitarse en las barras paralelas 9.

\section{FIGURA 2}

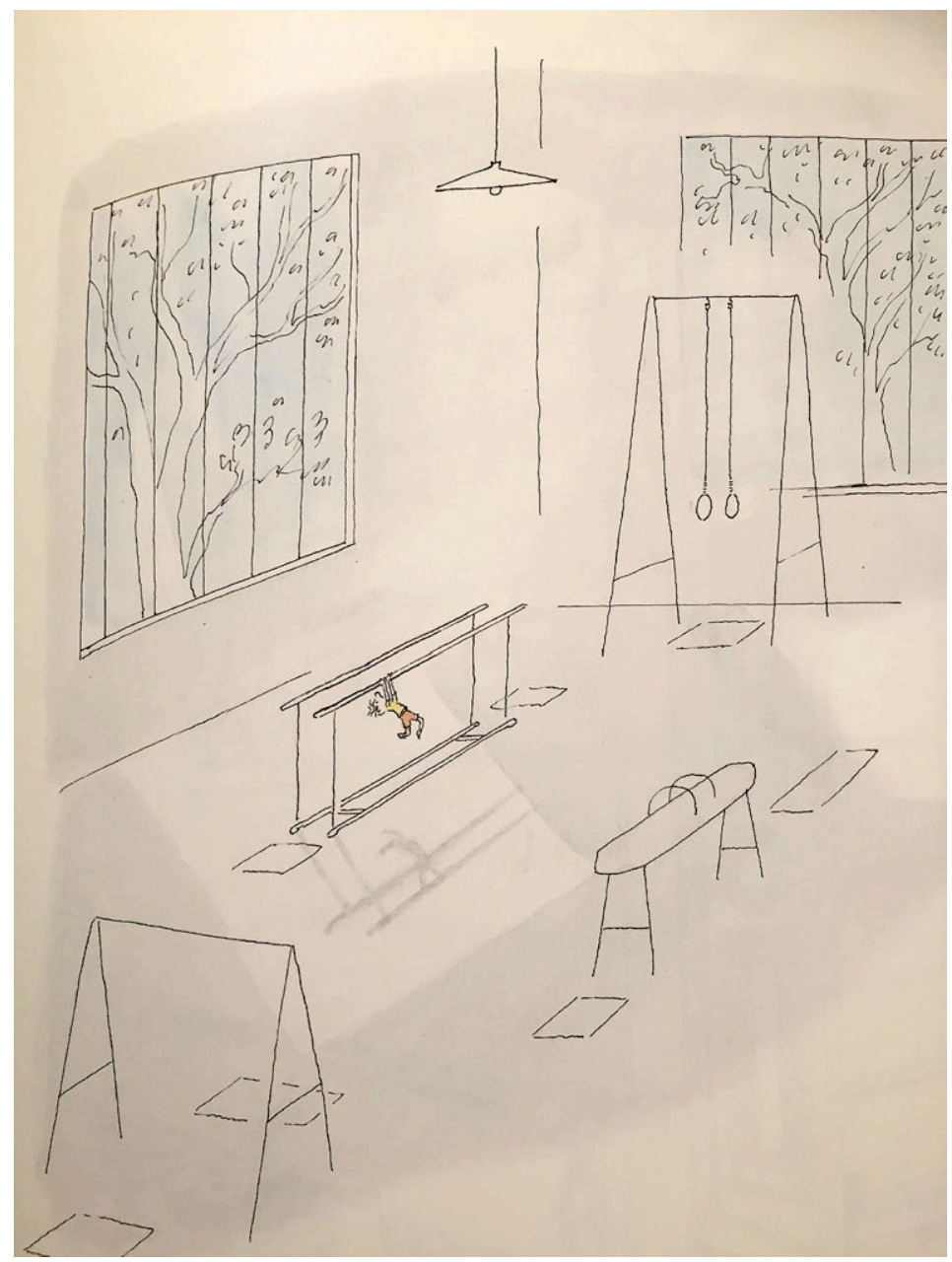

Jean-Jacques Sempé

In Enfances

Edition Galerie Martine Gossieaux 2004

9. Para un análisis kinésico de este dibujo y de otros tres de Sempé, ver Bolens (2014), consultable en: http://archive-ouverte.unige.ch/unige:74799. 


\section{FIGURA 3}

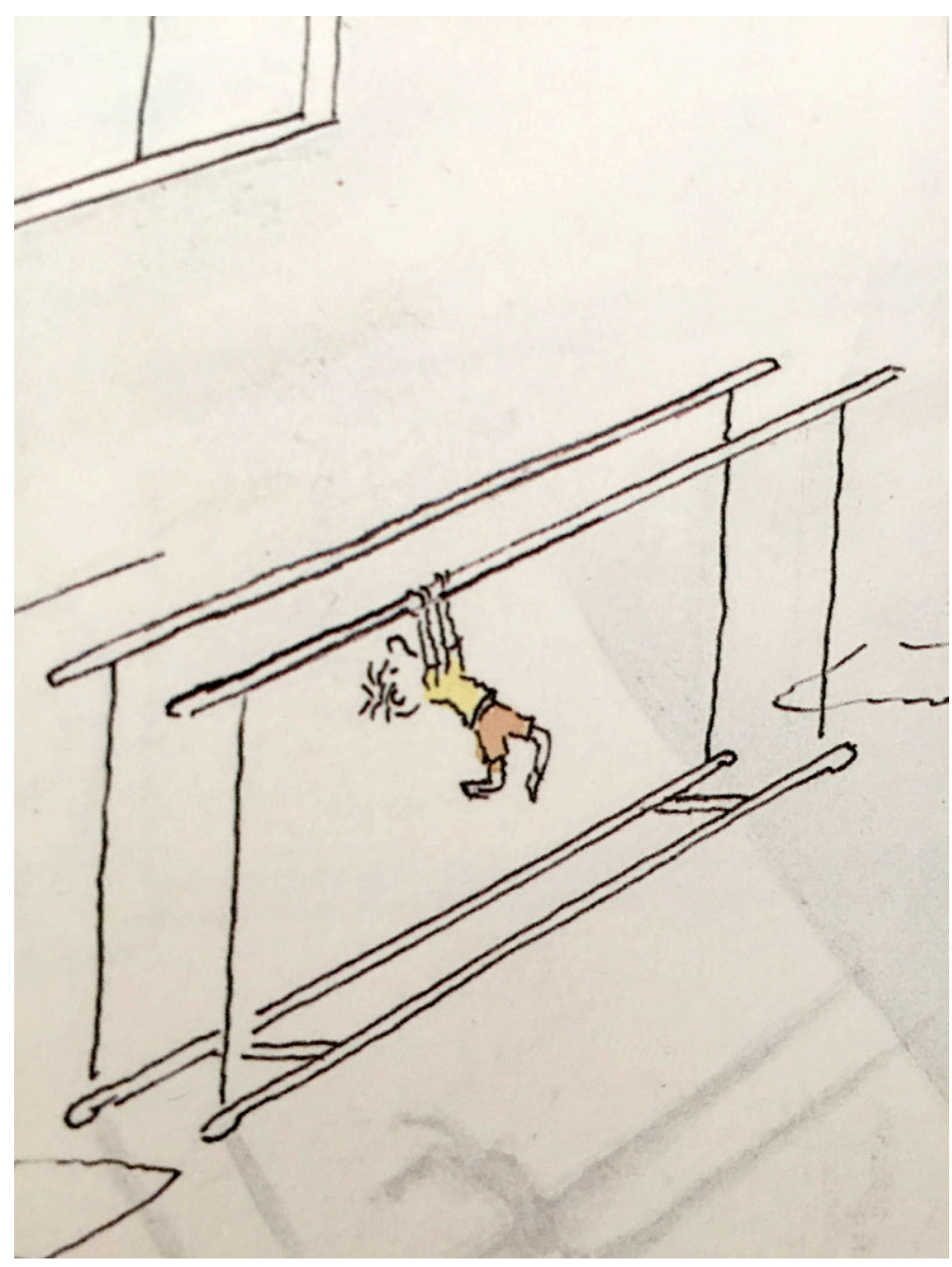

\section{Jean-Jacques Sempé} Enfances Detalle

El tono se compone del psiquismo y de la corporeidad, indefectiblemente ligados el uno al otro. Como toda vivencia corporal, se inscribe en una historicidad y una cultura que le dan un sentido variable. Pero, precisamente son esas variaciones las que nos permiten acceder en los textos (por volver a la literatura) a parámetros como el tono, a través de la introducción en el relato de lo que le señala. Hay tono muscular en el movimiento viviente, al que reaccionamos hoy como ayer. Mi objetivo consiste en tener en cuenta esta realidad fisiológica estable en el homo erectus y, al mismo tiempo, su inscripción en 
una realidad sociocultural histórica que, con su cambio constante, mediatiza siempre nuestro acceso a la realidad fisiológica del cuerpo. Los relatos literarios tienen la particularidad de activar por la ficción y el trabajo del lenguaje algunos parámetros que se encuentran en el corazón de las formas históricas de concebir el movimiento viviente. Para hacer que estos parámetros, emerjan, teniendo en cuenta que son actualizados por la ficción sin ser necesariamente conceptualizados y teorizados, es conveniente relacionar los textos entre sí.

\section{HUMOR E HIPERTEXTUALIDAD}

Según la definición de la hipertextualidad de Gérard Genette, «un hipertexto es un texto que deriva de otro por un proceso de transformación formal y/o temática (...) El hipertexto no es un a propósito, sino un a partir de su hipotexto» ${ }^{10}$. Don Quijote y Tristram Shandy son hipertextuales desde el momento en que se inscriben en una genealogía de textos múltiples de los que, sin embargo, se desmarcan claramente ${ }^{11}$. La deuda que reivindica Sterne con Cervantes se inscribe en esta filiación. Don Quijote y Tristram Shandy, las obras maestras de estos dos autores, son también hipertextuales como consecuencia de la enorme influencia que tuvieron sobre la literatura posterior. Su singularidad está construida sobre esta doble relación. Ahora bien, cuando aumenta en el lector la percepción del humor que contienen los textos ${ }^{12}$, el impacto de esta relación en el nivel de la recepción es notable. El reconocimiento por parte del lector de una relación hipertextual entre las obras acentúa el poder humorístico de éstas.

Mi método de análisis atiende a la dimensión hipertextual de un humor anclado en nuestro saber sensorimotor. No se trata de identificar recurrencias intertextuales de posturas, gestos o representaciones corporales ${ }^{13}$. Se trata más bien de percibir la manera en que el estilo kinésico de una obra -su peculiar manera de movilizar las dinámicas corporales de su relato y de sus personajes- dialoga con el estilo kinésico de otra obra. Se trata de acrecentar nuestra capacidad de percibir estos parámetros y de escuchar plenamente las voces que hablan de cuerpos en interacción -voces plasmadas en papel escrito hace cientos de años activadas ahora por el interés que podemos resentir al percibirlas.

10. Genette (2012: 24).

11. En Genette (1982: 201). Genette subraya el carácter hipertextual de Don Quijote y en particular su bien conocida relación con el llamado género de los «libros de caballerías» y de manera más precisa, de las obras tardías de este género como el Amadís de Gaula de Montalvo.

12 Genette (2012: 25): «Los hipertextos casi siempre establecen con sus autores una especie de contrato de hipertextualidad que les permite reconocer sus palabras, proporcionándoles así toda su eficacia.»)

13. Sobre el debate filosófico que tuvo lugar a propósito de la oposición entre representacionismo y enactivismo ver, por ejemplo, Chemero (2011); Noë (2006). Sobre la cognición situada ver Robbins y Aydede (2009). 
En la hipertextualidad de Don Quijote y Tristram Shandy los hipotextos (es decir, los textos que provocaron la escritura de esos nuevos textos) funcionan como palancas expresivas en el juego sensorimotor, cognitivo, epistemológico y afectivo puesto en marcha por los procesos del humor. Para abordar estos aspectos es preciso trabajar con herramientas complementarias que van desde el microanálisis kinésico hasta la contextualización social e histórica de los textos. Entre los focos kinésicos propuestos en estas páginas me concentraré en la inclusión en el relato de una modificación del tono corporal de los personajes. La literatura habla con frecuencia de modificaciones tónicas y de sus consecuencias sobre las interacciones humanas: es por ello necesario desarrollar instrumentos para observarlas y llevar a cabo su análisis.

\section{EL TONO CORPORAL EN LOS TEXTOS}

Vamos a considerar aquí dos pasajes, uno de Don Quijote y otro de Tristram Shandy, que describen un estado placentero provocado por un desarrollo cognitivo ligado a una actuación. En los dos ejemplos elegidos el relato habla de un placer cognitivo que modifica el tono corporal.

Por lo que se refiere al tono, la psicomotriz y psicóloga Suzanne Robert-Ouvray explica que «la motricidad es un agente de integración de datos corporales y cognitivos. El elemento fisiológico que permite esas integraciones es el tono muscular. El tono es la tensión que reside en el músculo cuando está inervado. Es la prueba de que los nervios han migrado hasta sus respectivos músculos; éstos se vuelven entonces reactivos y adquieren vida» ${ }^{14}$. Para Jean-Claude Coste el tono, «fenómeno nervioso muy complejo» que perdura mientras el cuerpo tiene vida, incluso cuando está inactivo, es «la trama de todos los movimientos» y «participa en todas las funciones motrices» tales como el equilibrio y la coordinación ${ }^{15}$. Robert-Ouvray añade que el elemento fisiológico es tan fundamental para nuestra manera de ser en el mundo que sus modificaciones, «ligadas a situaciones relacionales, permanecen a lo largo de toda la vida como nuestros sistemas de referencia y orientación afectiva hacia los demás $\rangle^{16}$. En efecto, como subraya Jacques Corraze, «todas las emociones reenvían a modificaciones tónicas» ${ }^{17}$.

Según el psicólogo André Bullinger, las funciones tónicas desempeñan un papel doble. Permiten la expresividad humana y al mismo tiempo sirven de «punto de apoyo para las funciones instrumentales» ${ }^{18}$. Son el lugar tanto de la relación motora, perceptiva y sensorial con el entorno, como de la expre-

14. Robert-Ouvray (1996: 31-32). Robert-Ouvray (2007). El tema central de esta obra es la importancia de la tonicidad en el desarrollo psicomotor del niño.

15. Coste (1994: 25).

16. Robert-Ouvray (1996: 96).

17. Corraze (2007: 71). Ver asimismo Cousin (1982/5: 363-385).

18. Bullinger (2013: 51). 
sión emocional en relación con ese mismo entorno. Por lo que se refiere a los flujos sensoriales, «participan de la relación tónico-postural y permiten situar al cuerpo en el espacio» ${ }^{19}$. Los flujos sensoriales son el flujo táctil, cuyo captador corporal es el conjunto del envoltorio cutáneo y, también, los flujos olfativos, sonoros y visuales. «Las respuestas del organismo a esos estímulos son tónicas y posturales $»^{20}$. Hay que añadir el flujo gravitatorio «constituido por la aceleración creada por las fuerzas de la gravedad y por las que suscitan los movimientos activos y pasivos del organismo». «Desde una perspectiva filogenética, el flujo gravitatorio ha determinado una parte importante de la arquitectura del esqueleto y de los músculos. Es esencial que los recursos tónicos y posturales estén presentes para componer con ese flujo» ${ }^{21}$.

Bullinger llama «sensibilidad profunda» a lo que «proporciona señales relativas al estado de tensión de los músculos, a la posición angular y a la velocidad de desplazamiento de las articulaciones. Esta sensibilidad profunda, que siempre está presente, se asocia a todas las señales que proceden de las diversas modalidades sensoriales $»^{22}$. Y añade:

Es la coordinación de estas dos series de señales, las procedentes de los flujos sensoriales y las que proceden de la sensibilidad profunda, la que permitirá constituir la función propioceptiva. Para que esta coordinación pueda realizarse es preciso poder detectar las concomitancias temporales entre dos series de eventos 23 .

Así pues, la función propioceptiva nace de la percepción de las concomitancias temporales que tienen lugar entre los eventos del ámbito de la sensibilidad profunda y los que proceden de los flujos sensoriales. Estos aspectos están en el centro de la perspectiva que trato de desarrollar en el análisis kinésico de la literatura, ya que permiten pensar la relación entre una vivencia corporal singular y su interfaz con una realidad perceptible compartida.

En otro lugar he propuesto utilizar con fines de análisis la triada kinésico, kinestésico y kinético. Por kinético entiendo todo aspecto motor relativo a las leyes de la física, como la ley de la gravedad, que se aplica de manera global tanto al mundo viviente como al no viviente. El sentido kinestésico corresponde a una realidad neurofisiológica del cuerpo vivo. Gracias al flujo sensorial kinestésico sentimos cada aspecto motor de nuestro cuerpo. Típicamente, gracias a esas informaciones sensoriales podemos activar nuestras manos sin mirarlas y saber exactamente qué movimiento estamos realizando ${ }^{24}$. Este sentido se entrena, por ejemplo, en una pianista, un bailarín o una cirujana. Gracias a ese flujo sensorial (que tiene mucho que ver con la sensibilidad profunda descrita por

19. Bullinger (2013: 81).

20. Bullinger (2013: 29).

21. Bullinger (2013:26).

22. Bullinger (2013: 82).

23. Bullinger (2013: 82).

24. Cf. Van Beers, Wolpert y Haggard (2002: 834-837). 
Bullinger) en conjunción con los otros flujos sensoriales, particularmente con el táctil, elaboramos permanentemente y de manera preconsciente nuestra experiencia fenomenológica de ser nuestro propio cuerpo, a la que reenvía el término propiocepción es decir, la percepción vital de que nuestro cuerpo es nuestro propio cuerpo. Para Suzanne Robert-Ouvray «(...) la propioceptividad como sentido kinestésico o sentido motor, y el tacto (o flujo sensorial táctil) son sistemas sensoriales constantes. Constituyen la base sensorial del sentimiento de la continuidad del vivir» ${ }^{25}$.

En fin, la kinesia reúne los dos términos precedentes (kinético y kinestésico) ampliando la perspectiva hasta una sensorimotricidad en interacción, tal como puede ser percibida, donde la realidad neurofisiológica se vincula no sólo a la realidad gravitacional y cualquier otra ley física, sino también a las instanciaciones socioculturales de los humanos que reaccionan a otros humanos y al mundo que los engloba. El campo de observación de la kinesia puede por ello ir desde el ínfimo sobresalto reflejo, fruto de la reacción a una manzana que cae de un árbol, hasta la expresión culturalmente codificada de unos gestos grandilocuentes realizados en el escenario de un teatro ante un público informado. Los fenómenos sensorimotores vinculan generalmente los tres parámetros: kinético (o físico), kinestésico (o sensorial) y kinésico (o interactivo y perceptivo). Distinguirlos con fines analíticos permite observar la naturaleza de sus impactos recíprocos.

En el abanico de los aspectos que constituyen cualquier acontecimiento kinésico, me propongo resaltar la cuestión de las variaciones tónicas. El tono es la manifestación permanente de la coordinación entre los flujos sensoriales y la sensibilidad profunda en relación con el entorno. Las variaciones tónicas se sitúan en el corazón de cualquier acontecimiento motor, tanto si se trata de una estricta inmovilidad como si se trata de un parpadeo subrepticio o de unas gesticulaciones desenfrenadas. Es posible permanecer inmóvil de muchas maneras, y sin que la postura cambie, pues la diferencia se sitúa en el nivel de la calidad tónica de la postura. Los grandes bailarines y los grandes actores son maestros en estas matizaciones.

La literatura describe a menudo modificaciones del tono para comunicar informaciones afectivas. Así, Cervantes no se refiere a una expresión facial para describir el júbilo de su héroe sino que reenvía a un aumento masivo del tono global del hidalgo mediante una descripción de naturaleza kinésica:

La del alba sería cuando don Quijote salió de la venta tan contento, tan gallardo, tan alborozado por verse ya armado caballero, que el gozo le reventaba por las cinchas del caballo. $(D Q \mathrm{I} \text {, iv, p. } 143)^{26}$.

Por su parte Sterne recurre a la comparación para hablar de una modificación tónica de su personaje Toby, tío de Tristram Shandy:

25. Robert-Ouvray (2007: 98).

26. Cervantes Saavedra (2006). Esta misma edición para todas las citas de este texto. Emplearé DQ para designar el título de la obra en las referencias de las citas. 
In the latter end of the third year, my uncle Toby perceiving that the parameter and semi-parameter of the conic section, angered his wound, he left off the study of projectiles in a kind of a huff, and betook himself to the practical part of fortification only; the pleasure of which, like a spring held back, returned upon him with redoubled force. (TS II, 4, p. 70)27

Al final del tercer año, mi tío Toby, al percibir que el parámetro y el semiparámetro de la sección cónica irritaba su herida, abandonó con una especie de resoplido el estudio de los proyectiles y se lanzó exclusivamente a la parte práctica de la fortificación; el consiguiente placer, como la liberación de un resorte comprimido, le embargó con redoblada fuerza (TS II, 4, p. 70) ${ }^{28}$.

Cervantes en la España del siglo XVII y Sterne en la Inglaterra del XVIII reenvían a una modificación tónica para significar un placer producido en sus personajes por un aumento de la inversión atencional. La inversión cognitiva y afectiva de Don Quijote tiene que ver con la actividad caballeresca de los relatos de ficción en los que se vio envuelto; el Tío Toby se aplica a la modelización de su «escena del crimen», es decir, el campo de batalla en el que fue herido para toda la vida. En los dos casos el texto reenvía a una modificación tónica, asociada a un placer a la vez físico y mental.

Por lo que se refiere a Don Quijote, su alegría explota por las cinchas de su caballo. Esta notable imagen oscila entre la metonimia y la analogía. Sugiere, en efecto, que el tono del caballero aumentó hasta tensionar las cinchas de Rocinante al límite de la explosión, pero también que el estado tónico de Don Quijote se asemeja a una correa de cuero estirada al máximo de su resistencia, hasta un punto de tensión que roza el desborde - de alegría. En cuanto al Tío Toby, el placer es el sujeto del verbo «to spring», al igual que en Don Quijote «el gozo» es el sujeto de «reventaba». De la misma manera que la alegría del hidalgo «le» estalla desde el cuerpo - ese cuerpo dual formado por el suyo y por el del caballo cinchado que le sostiene - el placer de Toby es el sujeto de un verbo que le asimila a un resorte comprimido al máximo y liberado de repente. En los dos casos la formulación es kinésica y se refiere a un artefacto: cinchas de caballo en el caso de Don Quijote, un resorte en el del Tio Toby. Los dos pasajes abren, por el estilo y el humor, una brecha referencial que prohíbe reducir la especificidad. En pocas palabras se inscriben en el cuerpo y en la Historia -Historia sociocultural de la caballería en un caso, historia de la filosofía mecanicista en el otro- ${ }^{29}$.

\section{Sterne (2004: 70).}

28. La expresión inglesa «in a huff» es intraducible literalmente al francés. Implica la producción de una bocanada de aire y el ruido que la acompaña en alguien que manifiesta una emoción provocada por una vejación, irritación y/o desprecio. (En español podría traducirse por resoplido, N. del T.).

29. Llevando más lejos la teoría del animal-máquina de Descartes, Julien Offroy de la Mettrie escribe que «el cuerpo humano es una máquina que monta ella misma sus propios resortes; viva imagen del movimiento perpetuo», La Mettrie (1981[1748]), Assoun (1981: 100). Según Alexis Tadié, Sterne no conocía la obra de La Mettrie, pero el contexto filosófico de estas cuestiones y los debates e intercambios a que dieron lugar debieron serle familiares. Tadié (2003: 54 n. 9). En el siglo XVIII el mecanicismo, el vitalismo, el racionalismo y el empirismo se disputaban el terreno. Cf. King (1978); Duchesneau (2012). 
El placer de los dos personajes tiene como objeto una actividad: la reactualización de las aventuras de los caballeros errantes para Don Quijote; la modelización de «su» campo de batalla para el Tío Toby. Esta actividad es la ocasión que motiva su gozo y pleasure. Es su resorte interno; actualiza su pulsión de vida, su drive, su alegría, su libido, su flow ${ }^{30}$, su apetencia, su stamina, su mojo o cualquier otro nombre que se le quiera dar. Sterne le llama el hobby horse. Lo que me interesa en los dos pasajes es la manera de comunicar ese placer vital y llamar la atención sobre la transformación tónica que lo manifiesta. Pero también que, en el nivel de la recepción de esas líneas, el lector elabora para comprenderlas una simulación perceptiva que procede de un saber sensorimotor que tiene que ver con el tono: en Don Quijote por una tonicidad acrecentada a través de un determinado tipo de movimientos y en el caso del Tio Toby por una desconexión tensional.

\section{EL ANÁLISIS LITERARIO DE LAS SIMULACIONES PERCEPTIVAS}

Una simulación perceptiva es aquello que elaboramos cognitivamente sobre la base de nuestro saber sensorimotor para producir un sentido a partir de una frase que se refiere a una acción, a una sensación o a una percepción, es decir, a cualquier información que requiera activar nuestra memoria sensorial y percepti$\mathrm{va}^{31}$. En los dos pasajes de Don Quijote y de Tristram Shandy es la condición necesaria no sólo para la comprensión de los enunciados sino, también, para el acceso a su humor. Dicho de otra forma, si el lector es sensible al humor de esas líneas es porque ha activado una simulación perceptiva sensorimotriz: kinética en lo que se refiere a la reactivación cognitiva de los movimientos de tracción (por las correas) y de compresión (para el resorte); kinestésica en el nivel de las sensaciones motrices asociadas a la tracción y a la compresión, y kinésica en el nivel perceptivo del estilo de los movimientos susceptibles de ser generados por las modificaciones tónicas producidas. Sin la simulación kinética, kinestésica y kinésica por parte del lector, los dos pasajes permanecen opacos. Es la activación cognitiva de ese saber sensorimotor la que permite comprender de qué manera el impacto kinestésico del placer experimentado por los dos personajes es comparable a las correas tensadas al límite o a un resorte comprimido al máximo y soltado después. El humor aparece cuando el acto cognitivo de la simulación perceptiva ilumina esas líneas. Imaginamos entonces, aunque sólo sea fugazmente, la clase de postura y tonicidad que marcan la kinesia de Don Quijote, cuya alegría es tan intensa que su placer se transmite de manera explosiva a las cinchas de su caballo.

30. Csikszentmihalyi (1990).

31. Markman, Klein y Suhr (2009); Pezzulo, Candidi, Dindo y Barca (2013: 270-290); Bolens (2012a) introduction; Bolens (2015: 115-125); Bolens (2012b: 143-156); Bolens (2014). 
«El gozo le reventaba por las cinchas del caballo» es una frase notablemente sintética y dinámica. Esta cualidad estilística provoca una simulación perceptiva eficaz por la que se comunican la intensidad y la velocidad del acontecimiento descrito, además del sentido codificado de los términos que forman la frase: el hidalgo explota de alegría y las cinchas de su caballo se resienten bruscamente. El lector, gracias a la fuerza estilística de la frase, sigue esta intensificación a través de la simulación perceptiva generada por una atenta lectura, ya que elaboramos simulaciones perceptivas como reacción al estilo mediante el cual el autor irriga el sentido que vehiculan las palabras. Es en el estilo donde el lenguaje se une al cuerpo y al saber que poseemos, a lo que hay que añadir que la simulación perceptiva de cada lector será la suya propia. En ningún caso se tratará de representaciones mentales comparables unas con otras como lo serían unas imágenes fijadas en el papel, en una película o en una pantalla. De lo que se trata es de una activación de la información en la forma en que es tratada por la inteligencia kinésica, configurada de manera cognitiva y sensorial por la comprensión dinámica del lector. A cada lector su Don Quijote tenso de alegría, pero a todos la información de un incremento tensional.

Querer observar esta etapa de la recepción de un texto - una entre otras, pero una etapa clave- no implica en modo alguno negar las variaciones sociales, epistemológicas, materiales o particulares en el momento en que fue escrito el texto o en el momento de nuestro acto de lectura. Se trata más bien de iluminar lo que a menudo constituye puntos ciegos en nuestra manera de leer un texto literario cuando las palabras, tratadas cognitivamente de forma pre-reflexiva, se trasladan luego directamente a conclusiones abstractas. Retener tan solo la idea de un Don Quijote contento en el pasaje que nos interesa no es suficiente. A través de esta idea, general y simple, se evacúa la literatura y, con ella, una mayor precisión. La literatura, por su trabajo sobre la lengua en relación con la corporeidad, es capaz de una complejidad que hay que reconocer y observar tomando en consideración nuestra inscripción histórica y la del texto.

En relación con lo anterior tomemos un breve ejemplo medieval de Geoffrey Chaucer, autor inglés del siglo XIV. En sus Cuentos de Canterbury, el personaje de ficción del propio Chaucer se pone en escena de forma humorística cuando forma parte de un grupo de peregrinos enzarzados en un concurso de cuentos en el camino que les conduce a Canterbury. Cuando a Chaucer le toca el turno de contar una historia divertida, resulta ser un narrador execrable y termina por ser interrumpido - él mismo, el autor de la obra maestra que estamos leyendo. Entre las razones del fracaso figura en cabeza de lista su notable capacidad de echar a perder las figuras de estilo. De tal forma que el personaje principal de su cuento, Sir Thopas, se enamora locamente (de nadie) simplemente al escuchar el canto de un tordo. Como reacción a esta súbita epifanía emocional espolea su caballo con tanta fuerza y durante tanto tiempo que el animal termina por sudar toda el agua de su cuerpo: «So swatte that men myghte him wrynge» ${ }^{32}$, 
sudaba tanto que unos hombres habrían podido escurrirlo (wring) - como se retuerce una prenda de vestir para extraerle el agua. Adentrarse plenamente en el texto explotando nuestras facultades cognitivas y sensorimotrices es una forma de tomar en consideración la voz del autor. En nuestro caso, es una simulación perceptiva la que nos permite acceder a la enloquecida imagen de un caballo escurrido como un trapo. Iluminar un instante esta activación cognitiva dinámica sirve para evidenciar nuestra participación en el texto en el momento de su recepción. Naturalmente cada lector tendrá una simulación idiosincrásica propia de su época, bien se trate de un vecino de Chaucer o de un helvético del siglo veintiuno. Lo cierto es que nuestro acceso a este discurso vendrá marcado por lo que este acceso mediatiza, es decir, por la realidad material de los médium empleados y la realidad de nuestra propia inscripción histórica, distante de varios siglos. Pero siendo esto cierto para cualquier acto de lectura, más vale poner este parámetro en su sitio y tener en cuenta en el campo observado nuestra propia participación cognitiva para con ello delimitar su alcance.

A todo ello hay que añadir todas las dimensiones culturales y epistemológicas por las que debemos aprehender las implicaciones de una parodia medieval de las novelas de sentimientos corteses, de la pasión amorosa, y del papel del caballo y la caballería en un autor inglés del siglo XIV. Esta vertiente complementa la precedente y la una no impide la otra, bien al contrario. El humor funciona por el trabajo de la lengua ligado a su contexto cultural. Así, cuando Chaucer todavía nos empuja a concebir un Sir Thopas que se lanza tan rápido al galope que resbala (glood) como lo haría la chispa que brota de un tronco en llamas («As a sparkle out of the bronde», I.905) tras haber colocado una torre en lo alto de su yelmo en la que prendió una flor de lis; si no experimentamos este doble movimiento -extrema rapidez linear de la chispa versus movimiento de vaivén de una torre ornamental encasquetada en la cabeza de un caballero al galope- no nos enteramos del texto. La referencia a una flor de lis fijada a la torre aumenta más aún la percepción del movimiento de vaivén impartido por el galope del caballo. En efecto, si la flor se ha fijado a una estructura cilíndrica es porque tiene un tallo; y si tiene un tallo su movimiento es flexible. La asociación paradójica de dos movimientos de intensidades y dinámicas contradictorias (extrema rapidez lineal y movimiento de vaivén flexible) fundamenta el humor de una elección figural soberbiamente fracasada. El lector puede apreciar simultáneamente la factura de este humor peculiar y percibir la ambigüedad política que, de paso, hace desempeñar al símbolo real francés un extraño papel evocado en un relato escrito durante la Guerra de los Cien Años, cuando se disputaba la legitimidad del rey de Francia entre distintas ramas genealógicas francesas e inglesas. No hay que elegir entre una atención a las simulaciones perceptivas provocadas por el texto y la contextualización histórica de las mismas. En este caso es más bien mediante nuestra atención a la simulación perceptiva, potencialmente provocada por el texto, por la que accedemos a su sobreentendido político. Basta con devolver el tallo a la flor de lis para que el símbolo real francés, desde el momento en que se coloca en la torre que corona un yelmo al galope, nos resulte maravillosamente cómico. 
A modo de conclusión, la figuralidad es un fenómeno discursivo que pone en marcha la atención mediante una exhibición de la enunciación. Mediatiza la referencia desplazándola, trasladándola -el ejemplo más claro es el de la metáfora a la que llamamos por su nombre latino, translatio-. Como la enunciación se exhibe entonces como separación, la atención del destinatario se fija de manera distinta, más focalizada sobre la forma de la información comunicada. Es esta forma - esta manera figural, este tropo- la que se encarga de iniciar una simulación perceptiva capaz de transmitir un tipo de información que una referencialidad directa tendría dificultades para comunicar.

La figuralidad desempeña un papel fundamental en la comunicación de datos complejos como la naturaleza exacta del estado tónico de personajes como Don Quijote en su caballo y el Tío Toby entregado a su pasión (en su hobby-horse). Porter Abbot subraya el impacto directo que la diferencia entre médiums puede tener sobre la manera de comunicar esta clase de información. La narración verbal ahonda esta diferencia cuando utiliza el lenguaje figural, especialmente la metáfora ${ }^{33}$. «A menudo, lo que es interno se escribe sobre el papel en lenguaje metafórico» ${ }^{34}$. Mientras que el cine proporciona el acceso a los datos visuales y sonoros tal y como han sido filmados y organizados en el montaje, en el lenguaje se traducen verbalmente esos mismos datos. Lo que la prosa narrativa pierde «en vitalidad física inmediata, visual y sonora, lo gana en flexibilidad figurativa $)^{35}$. Esta flexibilidad es notable en la descripción del tono modificado de Don Quijote y del Tío Toby y también en los tropos fracasados de Sir Thopas. Una traducción exclusivamente visual de esos momentos, bien por el trabajo de un actor en un escenario o en una película, en un dibujo o en una fotografía, podría representar los signos kinésicos de una disminución o de un incremento del tono de los personajes, pero perdería el efecto humorístico impartido por el registro figural de la expansión de un resorte al fin liberado o de las cinchas de Rocinante sometidas a una fuerte presión. De lo que se trata es de acercarnos a esta especificidad y a esta riqueza que nos permiten percibir una forma de intensidad propia de la literatura que se manifiesta por el estilo kinésico de las obras y por sus relaciones hipertextuales.

Traducción de José Luis Leal Maldonado 


\section{BIBLIOGRAFÍA CITADA}

Abbott, Porter (2008). The Cambridge Introduction to Narrative. Cambridge: Cambridge University Press. 2da. ed.

Bolens, Guillemette (2000). La Logique du corps articulaire. Les articulations du corps humain dans la littérature occidentale, Rennes, Presses Universitaires de Rennes, coll. «Histoire».

Bolens, Guillemette (2008). Le Style des gestes. Corporéité et kinésie dans le récit littéraire. Lausanne: Éditions BHMS.

Bolens, Guillemette (2012a). The Style of Gestures. Embodiment and Cognition in Literary Narrative. Baltimore: Johns Hopkins University Press.

Bolens, Guillemette (2012b). «Kinesthetic Empathy in Charlie Chaplin's Silent Films», en Kinesthetic Empathy in Creative and Cultural Practices. D. Reynolds y M. Reason (dir.). Bristol and Chicago: Intellect, pp.143-156.

Bolens, Guillemette (2014). «Les simulations perceptives et l'analyse kinésique dans le dessin et dans l'image poétique», Textimage. Revue d'étude du dialogue texte-image, 4, Consultable en: http://archive-ouverte.unige.ch/unige:74799.

Bolens, Guillemette (2015). «Les simulations perceptives dans la relation aux œuvres d'art littéraires», en Corps en scenes. M. Besson, C. Courtet, F. Lavocat, A. Viala (dir.) Paris: Editions du CNRS, pp. 115-125.

Bullinger, André (2013). Le développement sensori-moteur de l'enfant et ses avatars. Toulouse: Editions Érès.

Cave, Terence (2016). Thinking with Literature. Towards a Cognitive Criticism. Oxford: Oxford University Press.

Cervantes Saavedra, Miguel de (2006). El Ingenioso Hidalgo Don Quijote de la Mancha. F. Nieto (ed.). Madrid: Biblioteca Nueva.

Chaucer, Geoffrey (1987). The Canterbury Tales, «The Tale of sir Thopas», en The Riverside Chaucer. L. D. Benson (ed.) Oxford: Oxford University Press.

Chemero, Anthony (2011). Radical Embodied Cognitive Science. Cambridge (MA) and London: The MIT Press.

Corraze, J. (2007). «Le dialogue tonico-émotionnel à la lumière des connaissances actuelles», Entretiens de Psychomotricité. Paris: Expansion Formation et Editions.

Coste, Jean Claude (1994). La Psychomotricité. Paris: Presses Universitaires de France. 5ta. Edición.

Cousin Denise (1982). «L'investissement du dialogue tonique, sa place dans la genèse de la communication», Enfance. 35 (5), pp. 363-385.

Csikszentmihalyi, M. (1990). Flow: The Psychology of Optimal Experience. New York, London, Toronto: Harper Perennial Modern Classics.

Duchesneau, François (2012). La Physiologie des Lumières. Empirisme, modèles et théories. Paris: Classiques Garnier, coll. «Histoire et philosophie des sciences» 5.

Genette, Gérard (1982). Palimpsestes. La littérature au second degree. Paris: Seuil.

Genette, Gérard (2012). Des Genres et des ouvres, Paris, Seuil.

King, L.(1978). The Philosophy of Medicine. The Early Eighteenth Century. Cambridge (MA) and London: Harvard University Press.

La Mettrie, Julien Offray de (1981). L'Homme-machine [1748], P.-L. Assoun (ed.). Paris: Denoël/Gonthier.

Markman, K. D.; Klein, W. M. P. y Suhr, J.A. (dir.) (2009). Handbook of Imagination and Mental Simulation. New York: Taylor and Francis.

Noë, A. (2006). Action in Perception. Cambridge (MA) and London: The MIT Press.

Pezzulo, G.; Candidi, M.; Dindo, H. y Barca, L. (2013). «Action simulation in the human brain: Twelve questions», New Ideas in Psychology. 31, pp. 270-290. 
Robbins, P. and Aydede, M. (dir.) (2009). The Cambridge Handbook of Situated Cognition. Cambridge: Cambridge University Press.

Robert-Ouvray Suzanne (2007). Intégration motrice et développement psychique. Une théorie de la psychomotricité. Paris: Desclée de Brouwer. 2da. Edición.

Robert-Ouvray, Suzanne (1996). L'Enfant tonique et sa mère. Revigny sur Ornain: Hommes et perspectives/Editions Martin Media, coll. «Interfaces».

Rushdie, Salman (1991). «1,000 Days «Trapped Inside a Metaphor»», New York Times, 12 de diciembre de 1991, B8, col. 5.

Sterne, Laurence (2004). The Life and Opinions of Tristram Shandy, Gentleman. New York: The Modern Library.

Tadié, Alexis (2003). Sterne's Whimsical Theatres of Language. Orality, Gesture, Literacy. Aldershot: Ashgate.

Van Beers, R.; Wolpert, D. y Haggard P. (2012). «When Feeling Is More Important Than Seeing in Sensorimotor Adaptation», Current Biology, 12, pp. 834-837.

Wasserman, Edward A. y Zentall, Thomas R. (dir.) (2006). Comparative Cognition. Experimental Explorations of Animal Intelligence. Oxford: Oxford University Press.

Recibido: 20 de mayo de 2016

Aceptado: 24 de agosto de 2016 